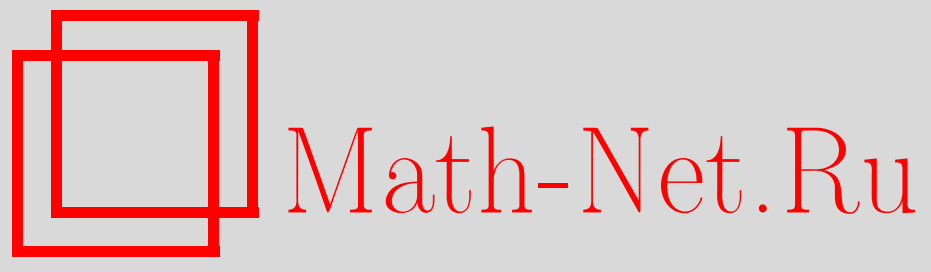

С. Н. Фёдоров, Арифметические условия мономиальности конечных групп, УМH, 2009, том 64, выпуск 1, 163164

DOI: https://doi.org/10.4213/rm9270

Использование Общероссийского математического портала Math-Net.Ru подразумевает, что вы прочитали и согласны с пользовательским соглашением http://www . mathnet.ru/rus/agreement

Параметры загрузки:

IP : 54.209 .52 .79

26 апреля 2023 г., 16:35:17

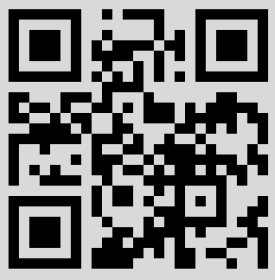




\section{Арифметические условия мономиальности конечных групп}

\section{С. Н. Фёдоров}

Конечная группа $G$ называется мономиальной или $\mathscr{M}$-группой, если для каждого ее неприводимого характера $\chi$ существует такой линейный характер $\lambda$ некоторой подгруппы $U \leqslant G$, что $\chi=\lambda^{G}$. Эквивалентное условие: матрицы каждого неприводимого представления $G$ (в некотором базисе пространства представления) мономиальны, т. е. имеют точно один ненулевой элемент в каждом столбце и каждой строке.

K. Такета [1] доказал, что $\mathscr{M}$-группы разрешимы. Обратное неверно, минимальный (по порядку) пример разрешимой немономиальной группы $\operatorname{SL}(2,3)$ порядка 24 $[2 ; \S 24.7 \mathrm{c}]$. Задача теоретико-групповой характеризации $\mathscr{M}$-групп значительно затруднена незамкнутостью класса $\mathscr{M}$-групп относительно взятия подгрупп. Как было показано Э. Дейдом, любая разрешимая группа изоморфно вкладывается в $\mathscr{M}$-группу (см. [3]). $\mathscr{M}$-группы, все подгруппы которых мономиальны, называют $\widetilde{\mathscr{M}}$-группами. Отметим, что метабелевы и сверхразрешимые группы являются $\widetilde{\mathscr{M}}$-группами.

Как известно, в теории конечных групп и их характеров существует некоторый параллелизм между результатами, касающимися классов сопряженных элементов группы, и результатами, касающимися ее неприводимых характеров. Однако часто оказывается, что условия, накладываемые на мощности классов сопряженности, жестче ограничивают вариации в строении группы, чем аналогичные условия на степени неприводимых характеров. Это подтверждают и предложения, приведенные ниже.

Некоторые обозначения: $g^{G}$ - класс сопряженных элементов группы $G$, содержащий $g \in G ; \operatorname{cs}(G)$ - множество мощностей классов сопряженных элементов группы $G$; $\operatorname{cd}(G)$ - множество степеней неприводимых характеров группы $G$.

Граф классов сопрлженных элементов $\Gamma(G)$ группы $G$ - это граф, вершины которого - все нецентральные классы сопряженных элементов группы $G$, вершины $g_{1}^{G}$ и $g_{2}^{G}$ соединены ребром тогда и только тогда, когда $\left(\left|g_{1}^{G}\right|,\left|g_{2}^{G}\right|\right) \neq 1$. Впервые дополнительный к описанному граф ввел Л. С. Казарин в [4]. Уже сам граф $\Gamma(G)$ исследовали с конца 1980-х годов Э. Бертрам, М. Герцог, А. Манн, Д. Чиллаг и др.

Аналогично определяется граф неприводимых характеров $\Delta(G)$ : вершины - нелинейные неприводимые характеры группы $G$, и вершины $\chi$ и $\psi$ соединены ребром, если $(\chi(1), \psi(1)) \neq 1$. Граф изучали О. Манц, В. Виллемс, Дж. Маквей, Д. Чиллаг и др.

Рассмотрим сначала условия арифметического характера на классы сопряженных элементов группы $G$, влекущие ее мономиальность или, в большинстве случаев, даже более сильные свойства.

(1) Если все элементы множества $\operatorname{cs}(G)$ - степени простого числа $p$, то группа $G$ нильпотентна.

Это нетрудно доказать, используя лишь тот факт, что мощность каждого класса сопряженности факторгруппы делит мощность некоторого класса самой группы.

(2) Если мощности всех нецентральных классов сопряженных элементов группы $G$ равны между собой, то группа $G$ нильпотентна [5].

(3) Если элементы множества $\operatorname{cs}(G)$ можно разбить на две непустые группы $\pi$-чисел и $\pi^{\prime}$-чисел для некоторого $\varnothing \neq \pi \varsubsetneqq \pi(G)$ (в частности, если они являются степенями простых чисел), то группа $G$ нильпотентна или метабелева.

Здесь, кроме (1) и (2), используем результаты [6] о случае несвязного графа $Г$.

(4) Если все элементы множества $\operatorname{cs}(G)$ - последовательные натуральные числа oт 1 до $k$, то $k \leqslant 3$ и группа $G$ метабелева.

Это следствие применения результатов [2; § 32.10а] и [6].

(5) Если все элементы множества $\operatorname{cs}(G)$ свободны от квадратов, то группа $G$ сверхразрешима [7]. 
(6) Если граф классов сопряжсенных элементов $\Gamma(G)$ группы $G$ не содержит полного подграфа с четырьмя вериинами, то $G-\widetilde{\mathscr{M}}$-группа.

Доказательство использует основную теорему из [8]. Заметим, что нельзя усилить вывод этого утверждения ни до сверхразрешимости, ни до метабелевости - примером тому служит группа $S_{4} \operatorname{c~} \operatorname{cs}\left(S_{4}\right)=\{1,3,6,8\}$ (6 входит с кратностью 2$)$. Если потребовать, чтобы $\Gamma(G)$ не содержал полного подграфа с пятью вершинами, то можно показать, что в этом случае либо $G-\widetilde{\mathscr{M}}$-группа, либо $G \cong A_{5}$.

Заметим в качестве следствия, что в условиях утверждений (1)-(6) группа $G$, очевидно, мономиальна.

Перейдем теперь к вопросу о возможных аналогах данных утверждений в случае степеней неприводимых характеров вместо мощностей классов сопряженности.

$\left(\mathbf{1}^{\prime}\right)$ Если все элементы множества $\mathrm{cd}(G)$ - степени простого числа $p$, то группа $G$ мономиальна.

Группа $A_{4} \cong \operatorname{PSL}(2,3)$ удовлетворяет условию, но не является сверхразрешимой. Можно также построить подходящий пример неметабелевой группы.

$\left.\mathbf{( 2}^{\prime}\right)$ Если степени всех степеней нелинейных неприводимых характеров группљ $G$ равны между собой, то группа $G$ метабелева $[2 ; \S 27.1]$.

$\left.\mathbf{( 3}^{\prime}\right)$ Если все элементы множества $\mathrm{cd}(G) \backslash\{1\}$ являются простыми числами, то группа $G$ разрешима $[2 ; \S 31.1]$.

Если, как в (3), ослабить условие до степеней простых чисел, то вывод будет неверен: например, $\operatorname{cd}\left(A_{5}\right)=\left\{1,2^{2}, 3,5\right\}$. С другой стороны, вывод утверждения усилить нельзя: немономиальная группа $G=\operatorname{SL}(2,3)$ имеет $\operatorname{cd}(G)=\{1,2,3\}$. Этот же пример не позволяет сформулировать адекватный аналог утверждения (4) (о группах с последовательными степенями неприводимых характеров см. [9]).

$\left(5^{\prime}\right)$ Если элементы множества $\mathrm{cd}(G)$ свободны от квадратов, а силовские р-подгруппы группы Фиттинга $F(G)$ не имеют сечений, изоморфных группе кватернионов порядка 8 для $p=2$ и экстраспециальным группам порлдка $p^{3}$ экспоненты $р$ для нечетных $p$, то либо (а) $G$ мономиальна; либо (b) $G \cong A_{7} \times H$, где $H$ - $\mathscr{M}$-группа, $u$ тогда $\operatorname{cd}(G)$ содержит множество $\{1,6,10,14,15,21,35\}$.

В основе доказательства этого факта лежат результаты из работы [10].

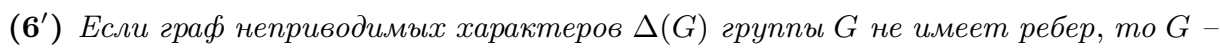
нильпотентна или метабелева.

Такой будет, например, несверхразрешимая группа $A_{4}$, имеющая единственный нелинейный неприводимый характер. Если граф $\Delta(G)$ не содержит треугольников, то в общем случае $G$ даже не разрешима: $\Delta\left(A_{5}\right)$ состоит из двух соединенных ребром вершин, соответствующих характерам степени 3 , и двух изолированных вершин.

\section{Список литературы}

[1] K. Taketa, Proc. Imp. Acad., 6:2 (1930), 31-33. [2] B. Huppert, Character theory of finite groups, de Gruyter Exp. Math., 25, de Gruyter, Berlin, 1998. [3] G. M. Seitz, Math. Z., 110:2 (1969), 101-122. [4] Л. С. Казарин, Изв. вузов. Матем., 1981, №7, 40-45. [5] N. Itô, Nagoya Math. J., 6 (1953), 17-28. [6] E. A. Bertram, M. Herzog, A. Mann, Bull. London Math. Soc., 22:6 (1990), 569-575. [7] D. Chillag, M. Herzog, J. Algebra, 131:1 (1990), 110-125. [8] A. Moretó, G. Qian, W. Shi, Arch. Math. (Basel), 85:2 (2005), 101-107. [9] B. Huppert, Forum Math., 1:2 (1989), 167-183. [10] B. Huppert, O. Manz, Arch. Math. (Basel), 45:2 (1985), 125-132.

\section{H. Фёдоров (S. N. Fedorov)}

Московский государственный университет им. М. В. Ломоносова

E-mail: Sergey.Fedorov@mail.ru
Представлено А. В. Михалёвым Принято редколлегией 07.12.2008 